\title{
Substance-P in symptomatic mediopatellar plica as a predictor of patellofemoral pain
}

\author{
RUI HE, LIU YANG, GUANGXING CHEN, LIN GUO and YING PEI \\ Center for Joint Surgery, Southwest Hospital, The Third Military Medical University, Chongqing 400038, P.R. China
}

Received July 16, 2015; Accepted September 8, 2015

DOI: $10.3892 / b r .2015 .531$

\begin{abstract}
The present study aimed to investigate the expression of a neuropeptide of symptomatic mediopatellar plica (MP) to prove that there was a significant correlation between the expression of substance-P (SP) in MP and patellofemoral pain. The specimens of synovial plica were obtained by knee arthroscopy. Semi-quantitative analysis was used to investigate the expression of SP, and compared the innervation density of SP among the three groups: Blank control (asymptomatic plica), positive control (symptomatic plica with other injuries) and experimental (symptomatic plica without other injuries) groups by the paired t-test, one-way analysis of variance and Student-Newman-Keuls test in 60 patients. The expression levels of SP nerve fibers in the superior plica were $24.60 \pm 26.17,117.36 \pm 73.62$ and $59.06 \pm 44.06$ in the blank control, positive control and experimental groups, respectively. The density of nerve fibers in medial plica was $23.23 \pm 18.41$ in the blank control group, $268.00 \pm 71.60$ in the positive control group and $255.44 \pm 87.91$ in the experimental group. The density of nerve fibers of MP was higher compared to that of the superior plica. The density was highest in the positive control group, and lowest in the blank control group in MP. There was a close correlation between the density of SP expression and the degree of patellofemoral pain. The SP expression intensity has an important role in the incidence of patellofemoral pain and is responsible for the pathogenesis of symptomatic MP.
\end{abstract}

\section{Introduction}

Mediopatellar plica (MP) in the knee is the synovial structure of the knee joint cavity that is derived from embryonic mesenchymal tissues without full absorption during embryonic development. In arthroscopy and autopsy studies, the

Correspondence to: Professor Liu Yang, Center for Joint Surgery, Southwest Hospital, The Third Military Medical University, 30 Gaotanyan Street, Chongqing 400038, P.R. China

E-mail: jointsurgery@163.com

Abbreviations: MP, mediopatellar plica; SP, substance-P

Key words: mediopatellar plica, immunohistochemistry, substance-P, patellofemoral pain incidence rate of MP was $19-70 \%$, of which $1 / 8$ to $1 / 10$ had pathological MP with pain in the patellofemoral joint (1). Typical symptoms include painful locking within the joint and chronic patellofemoral intermittent dull pain, which became severe in up and downstairs movements and when sedentary. These symptoms are not usually associated with knee effusions or swelling (2-4).

The anatomic structure, and physiological and pathological mechanisms in MP that may lead to pain remain to be elucidated. The possible reasons for causes of patellofemoral pain include: i) MP may have changed the normal biomechanic distribution of the patellofemoral joint and physiological load of the patellofemoral joint cartilage, leading to the stress increase of partial articular cartilage and subchondral bone, which resulted in localized cartilage damage and pain. The nociceptors that were isolated from cartilage under the patella bone can provide a theoretical basis for this hypothesis (5). ii) Patellofemoral pain may come from the joint capsule, subchondral bone, patellar fat pad, retinaculum, cartilage, the patellar tendon and synovial plica (5-8). Stimulated by trauma and other chronic inflammatory factors, localized hemorrhage may occur in the MP followed by fibrosis, thickening of the synovial tissue, inflammation and long-term recurrent chronic impact, which leads to patellofemoral pain (9). Substance-P (SP) is an undecapeptide that functions as a neurotransmitter and a neuromodulator. This peptide is widely distributed throughout the central and peripheral nervous systems. There is substantial evidence that neuropeptide SP is involved in neurogenic inflammation. Macrophages can be activated by SP, provoking the release of inflammatory compounds. The release of these chemical mediators is crucial for inflammatory response. SP appears to have a major role in the development and maintenance of pain and inflammation, and high levels of the neuropeptide are associated with pain and persistent inflammation.

Therefore, we hypothesized that the increase of the distribution of nerve fibers in symptomatic MP and its expression of neuropeptide had a close correlation to the occurrence and sustenance of pain. In the present study, immunohistochemistry was used to investigate SP expression levels in MP. Semi-quantitative analysis was utilized to compare the expression difference of SP in asymptomatic plica and symptomatic plica with patellofemoral pain, and the association between SP expression and patellofemoral pain was discussed. 
Table I. Sakakibara classification and demography.

\begin{tabular}{|c|c|c|c|c|c|c|}
\hline \multirow[b]{2}{*}{ Group } & \multicolumn{4}{|c|}{ Sakakibara classification } & \multirow{2}{*}{$\begin{array}{l}\text { Age, mean } \pm \text { standard } \\
\text { deviation (range) }\end{array}$} & \multirow[b]{2}{*}{ Male/female } \\
\hline & $\mathrm{A}$ & $\mathrm{B}$ & $\mathrm{C}$ & $\mathrm{D}$ & & \\
\hline Blank control & 7 & 9 & 4 & 0 & $34.8 \pm 12.0(14-58)$ & $8 / 12$ \\
\hline Positive control & 0 & 7 & 9 & 4 & $33.8 \pm 8.8(17-52)$ & $10 / 10$ \\
\hline Experimental & 1 & 5 & 11 & 3 & $28.9 \pm 7.1(17-39)$ & 9/11 \\
\hline Total & 8 & 21 & 24 & 7 & $32.5 \pm 9.8(14-58)$ & $27 / 33$ \\
\hline
\end{tabular}

\section{Materials and methods}

Reagent. The rabbit anti-human SP polyclonal antibody/fluorescein isothiocyanate labeled goat anti-rabbit immunoglobulin G (ZsBio, Beijing, China) and 4,6-diamidino-2-phenylindole (DAPI) (Beyotime Co., Nantong, China) were used.

Inclusion criteria and group division. i) The blank control group included 20 patients (20 knees) who were diagnosed with only lateral discoid meniscus and MP without patellofemoral joint pain; and ii) the positive control group included 20 patients (20 knees) who were diagnosed with synovial plica syndrome preoperatively, concomitant with chondromalacia patella intraoperatively and femoral condyle cartilage injuries. iii) The experimental group was the 20 patients ( 20 knees) who were diagnosed with MP and no other concomitant disorders in the joint.

Exclusion criteria. Patients with systemic diseases, such as diabetes, systemic lupus erythematosus, rheumatoid arthritis and other chronic inflammatory conditions, in the knee were excluded.

The study was approved by the Institutional Review Board and Ethics Committee at The Center for Joint Surgery (Southwest Hospital, The Third Military Medical University, Chongqing, China). All the patients provided signed informed consent.

Preoperative and postoperative pain score evaluation. The visual analogue scale (VAS) was used to evaluate the patients' patellofemoral joint pain preoperatively and postoperatively. Patients were followed for $12 \pm 3.9$ months (range, 6-19 months). The VAS 3-month postoperative results were obtained to compare with the preoperative scores.

Arthroscopy and specimen preparation. A total of 60 patients (60 knees) underwent arthroscopy with an arthroscope that was equipped with a digital colored three-chips endoscopic camera system inside (Smith \& Nephew, Andover, MA, USA). The procedure was completed by the same arthroscopic surgeon. The diagnosis of symptomatic MP can be confirmed by the presence of pathological disorders in the arthroscope (Fig. 1) (10,11).

The specimens were obtained from the tissues in the basilar section of the suprapatellaris plica and MP of each patient.
Immunohistochemical study. The specimens were refrigerated at $4^{\circ} \mathrm{C}$, frozen in liquid nitrogen, embedded by a mixture of polyethylene glycol and polyvinyl alcohol (optimal cutting temperature compound) and sliced. The slices were $8 \mu \mathrm{m}$. Permeability was performed in phosphate-buffered saline (PBS) with $0.1 \%$ (volume fraction, V/V) Triton $\mathrm{X}-100$ for $30 \mathrm{~min}$ and sealed for $15 \mathrm{~min}$ with $5 \%$ goat serum (V/V). The slice was incubated in PBS and rabbit anti-human SP (ZA-0235) 1:50 (V/V). The section was incubated overnight in a damp environment at $4^{\circ} \mathrm{C}$. Following this, it was rinsed with $0.01 \mathrm{~mol} / 1 \mathrm{PBS}$ three times, for $5 \mathrm{~min}$ each time. The fluorescent secondary antibody was added to the procedure. The marker was fluorescein isothiocyanate goat-anti-rabbit (ZF-3011) 1:50 (V/V) and the sample was incubated at the constant temperature of $37^{\circ} \mathrm{C}$. Following rising with $0.01 \mathrm{~mol} / 1$ PBS three times, 5 min each time, the sample was dyed with DAPI for $1 \mathrm{~min}$ and $50 \%$ glycerine for mounting. Samples were observed with a fluorescence microscope.

Semi-quantitative analysis. Image analysis software Olympus DP Controller and DP Manager (Olympus Corp., Toyko, Japan) was used with 400-power random observation for 50 visual fields. All the immunoreactive fibers were numbered and the density of the nerve fibers was calculated (fibers $/ \mathrm{cm}^{2}$ ). The data collection was completed by two pathological physicians in a double-blind manner.

Statistical analysis. SAS JMP6.0 software (SAS Institute, Inc., Cary, NC, USA) was used to analyze the statistical data, with the size of the test set at $\alpha=0.05$. Nerve density differences between the groups were compared by the method of analysis of variance and Student-Newman-Keuls test. Nerve density differences between the MP and suprapatellar plica were compared by the paired t-test. The VAS score differences of the experimental and positive control group preoperative and 3 months postoperative were analyzed by the Wilcoxon rank sum test. The association between the preoperative VAS pain score and the SP fiber density of MP were analyzed by the Spearman's correlation.

\section{Results}

Changes in structure between the groups. The standard Sakakibara (12-14) taxonomy was applied in MP arthroscopic classification criteria, as shown in Fig. 2.

Pathological changes occurred between groups. The plica structure widened significantly more compared to the blank 

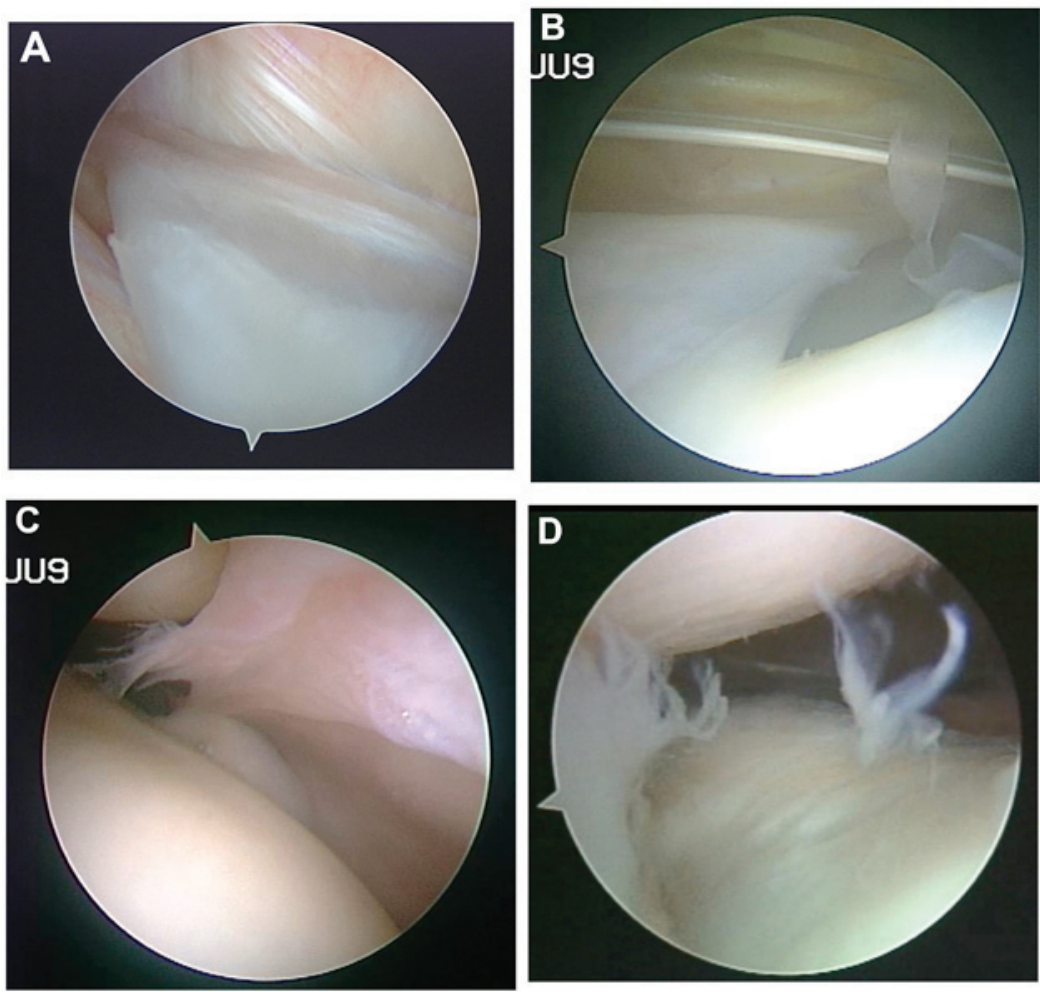

Figure 1. Diagnosis of symptomatic MP in arthroscope. (A) Plica hypertrophied with its breadth over $1 / 2$ to $1 / 3$ of femoral condyle. (B) Plica ischemia; thicken and stiffen, and fibration with elasticity loss. (C) Plica hyperemia and edema. (D) Plica reaching into the joint space in movement; cartilage becomes crude and softens, exfoliates or an indentation-like wear is formed. MP, mediopatellar plica.
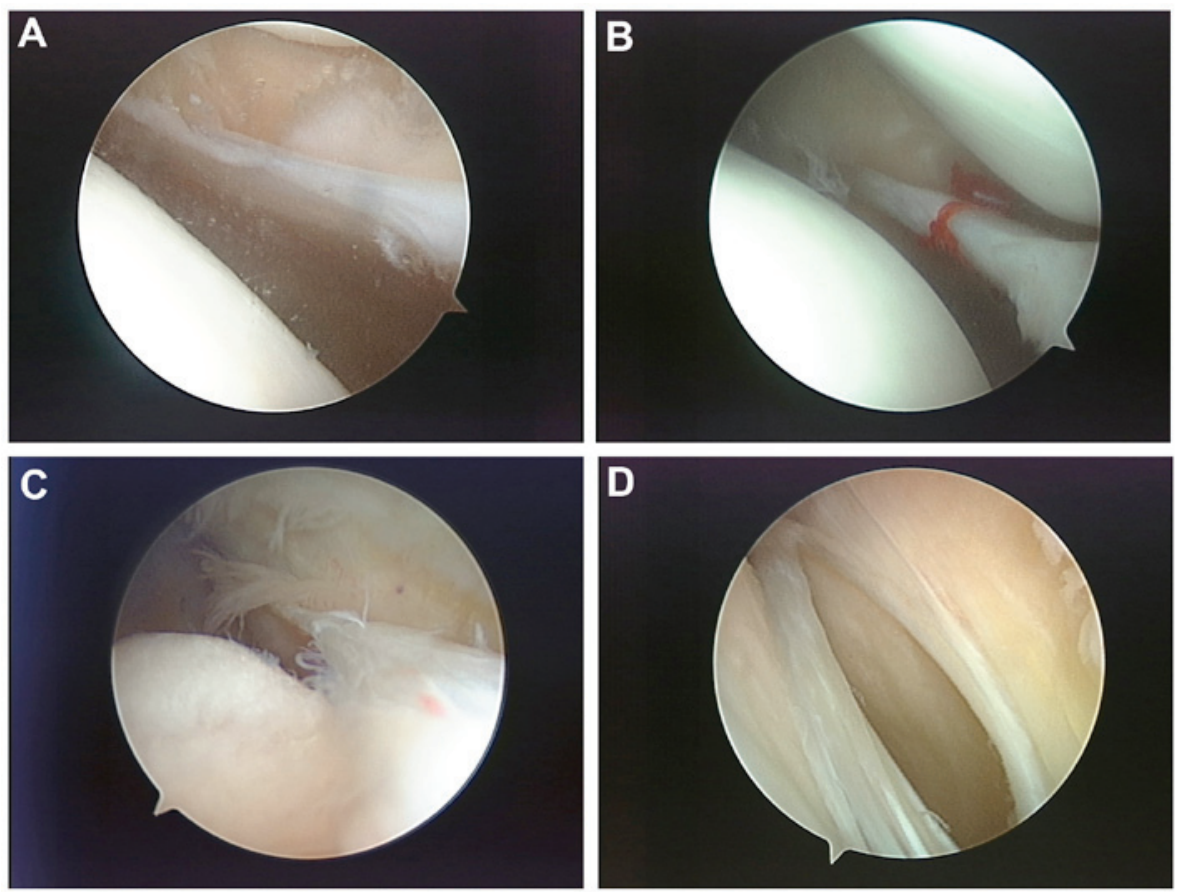

Figure 2. MP arthroscopic classification criteria. (A) Type A: The plica form a small membrane-like bulge on the medial wall (cord-like). (B) Type B: Plica enlargement, with its breadth over $1 / 2$ to $1 / 3$ of medial femoral condyle (shelf-like). (C) Type C: A large shelf that covers the anteromedial femoral condylar surface. (D) Type D: The same shape as type C, with bucket handle tears in the middle of the plica, which had a double insertion on the medial wall. MP, mediopatellar plica.

control (types C and D) in 31 cases, fibrous cord-like changes occurred in 22 cases, ischemic pale hypertrophy in 11 cases, and extrusion-forming flaps in 13 cases, respectively (Table I).
No significant statistical difference was observed in gender $(\mathrm{P}=0.817)$ and age $(\mathrm{P}=0.135)$ among the blank control, experimental and the positive control groups. Type $\mathrm{C} / \mathrm{D}$ in the 
Table II. Staining SP nerve fiber density of suprapatellar plica and MP.

\begin{tabular}{|c|c|c|c|c|}
\hline Group & Case, $\mathrm{n}$ & Suprapatellar plica & MP & Statistic \\
\hline Blank control & 20 & $24.60 \pm 26.17$ & $23.23 \pm 18.41$ & $\mathrm{t}=0.14$ \\
\hline Positive control & 20 & $117.36 \pm 73.62$ & $268.00 \pm 71.60$ & $\begin{array}{l}\mathrm{P}=0.8884 \\
\mathrm{t}=10.51\end{array}$ \\
\hline Experimental & 20 & $59.06 \pm 44.06$ & $255.44 \pm 87.91$ & $\begin{array}{l}\mathrm{P}=0.0056 \\
\mathrm{t}=13.13\end{array}$ \\
\hline Statistics & & $\begin{array}{l}\mathrm{F}=11.27 \\
\mathrm{P}<0.001\end{array}$ & $\begin{array}{l}F=47.70 \\
P<0.001\end{array}$ & $\mathrm{P}=0.0013$ \\
\hline
\end{tabular}

Staining SP nerve fiber density in MP: Positive control group was higher compared with the experimental group, $\mathrm{P}=0.043$; experimental group was higher compared with the blank control group, $\mathrm{P}<0.001$; positive control group was higher compared with the blank control group, $\mathrm{P}<0.001$. MP, mediopatellar plica; SP, substance-P.

Table III. VAS pain evaluation results.

\begin{tabular}{lccrr}
\hline Group & Case, $n$ & Preoperative & 3-month postoperative & Statistics \\
\hline Experimental group & 20 & $61.35 \pm 22.36$ & $11.78 \pm 3.77$ & $\mathrm{~W}=210, \mathrm{P}=0.0027$ \\
Positive control group & 20 & $68.45 \pm 16.39$ & $12.96 \pm 5.31$ & $\mathrm{~W}=213, \mathrm{P}=0.0095$ \\
Statistics & & $\mathrm{W}=373, \mathrm{P}=0.371$ & $\mathrm{~W}=384, \mathrm{P}=0.481$ & \\
\end{tabular}

No significant difference was observed in the preoperative pain score of the experimental and positive control groups. Postoperative pain scores were significantly lower. VAS, visual analogue scale.

experimental group was higher compared to the blank control group $(\mathrm{P}=0.001)$, and there was no significant difference between the experimental and positive control groups $(\mathrm{P}=0.736)$.

Distribution of SP fibers. Immunohistochemical staining revealed a positive performance of SP in MP (magnification, $x 400)$ as shown in Fig. 3.

Semi-quantitative data analysis of SP staining is shown in Table II. Staining SP nerve fiber density in suprapatellar plica revealed that the positive control group was higher when compared to the experimental and blank control groups, $\mathrm{P}<0.001$; however, there was no difference between the experimental and blank control groups.

VAS pain evaluation. The VAS pain evaluation results are shown in Table III. No significant difference was observed in the preoperative pain score of the experimental and the positive control groups. Postoperative pain scores were significantly lower.

Association of VAS pain evaluation and density of SP fibers of $M P$. The correlation between VAS pain score and innervation density of SP in 40 patients in the experimental and positive control groups was analyzed. The results showed a positive correlation between SP positive nerve density and pain scores ( $\mathrm{r}=0.4612, \mathrm{P}=0.0027)$.

\section{Discussion}

In previous studies, including those by Broom andFulkerson (15), Jackson et al (16) and Shahriaree and Nottage (17), the contact between mediopatellar and femoral condyle was reported to not cause pain directly, however, types A and B, with smaller morphological plica, can cause patellofemoral pain. There is no direct correlation between the occurrence of the patellofemoral pain and plica morphology. However, the basic research with regards to its clinical causes remains to be elucidated.

Currently, an increasing number of studies have focused on the study of neurophysiological changes of soft tissues in the knee joint to explain a variety of pathological changes and functional abnormalities $(5,18,19)$. Certain studies have reported that the level of neuropeptide nerve fibers in patients with anterior knee pain rose significantly in the tissues of retinaculum and fat pads (7). In the previous studies it has been confirmed that SP is an important neurotransmitter of pain, and the material basis for the conduction of pain, whose level is the indicator of the pain degree experienced by the patient in the knee joint and soft tissue (20-23).

An objective pain assessment was affected by patient attributes, including psychological factors and individual differences, so therefore, the experiments were designed by taking the specimens from the same patient, which can reduce the influence on the experimental data caused by individual differences for the feelings of pain. In the present study, the SP expression levels in patients with MP and suprapatellar plica were compared within the same group. The study found that in the blank control group, there was no significant difference in the SP fibers distribution. There was also no pain symptom in MP and in suprapatellar plica. In the experimental and positive control groups, the SP expression levels in MP were significantly higher compared to the levels of suprapatellar plica. 

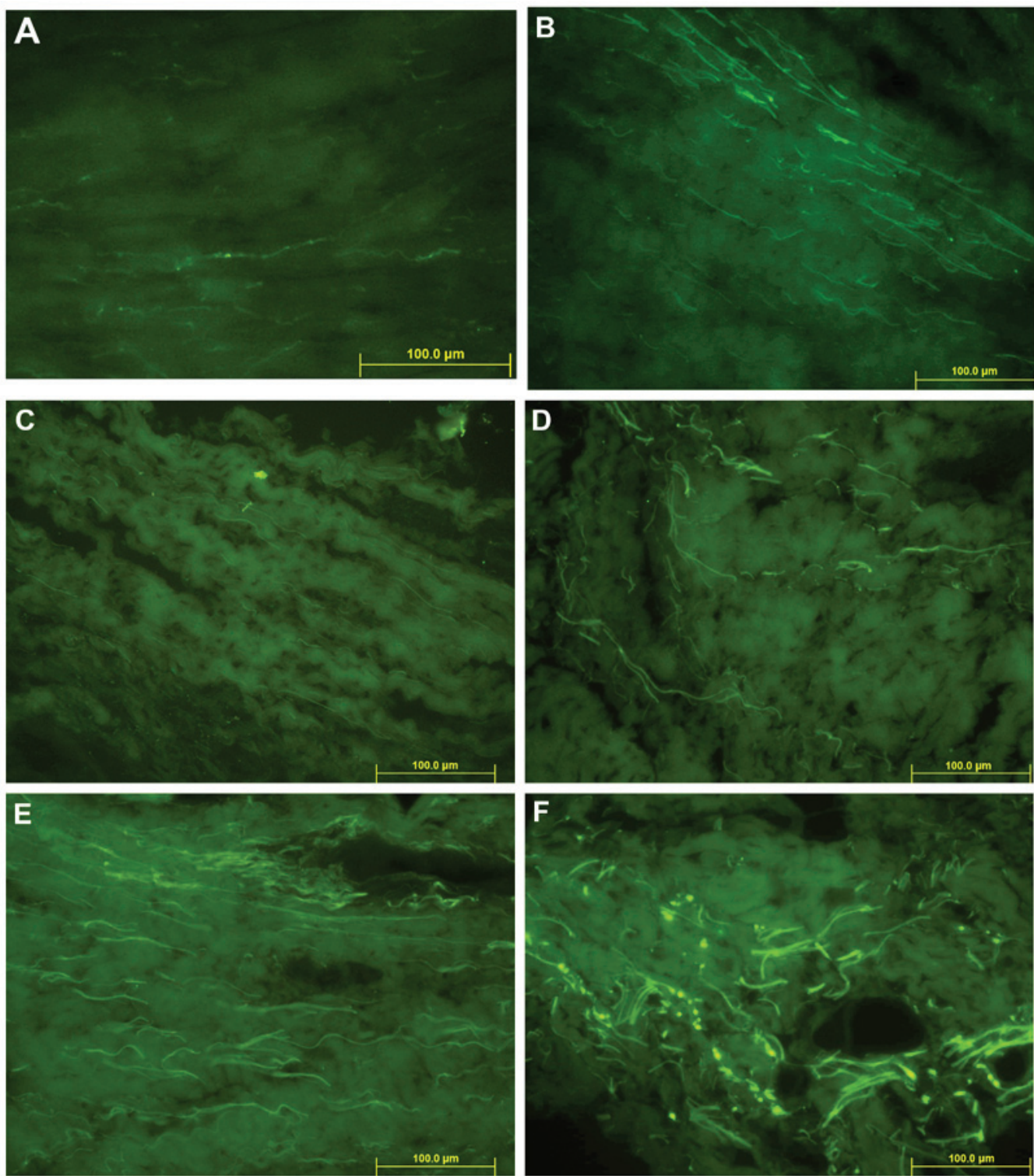

Figure 3. Immunohistochemical staining positive performance of SP in MP (magnification, x400). (A) Free nerve endings (green fluorescent fibers) were observed in the suprapatellar plica of the blank control group, scattered in the collagen fibers. (B) Free nerve endings (green fluorescent fibers) were visible in the suprapatellar plica of the positive control group, distributed in the collagen fibers background. (C) A small amount of free nerve endings (green fluorescent fibers) were observed in the suprapatellar plica in the experimental group; scattered. (D) Free nerve endings (green fluorescent fibers) were visible in the MP of the blank control group, scattered in the collagen fibers. (E) Patients with MP of the positive control group, showing free nerve endings (green fluorescent fibers) with a significant increase; intensive. (F) Patients with MP in the experimental group, showing free nerve endings (green fluorescent fibers), distributed in the collagen fibers. Neural network structure was observed in the peripheral vascular wall. MP, mediopatellar plica; SP, substance-P.

There was no suprapatellar bursa effusion, inflammation or pain in the selected specimens. A high degree of consistency existed between the increase of density of SP fibers in MP and the aggravated patellofemoral pain.

Arthroscopic resection of symptomatic MP can significantly alleviate and eliminate the symptoms of patellofemoral pain, which has been confirmed by a number of previous studies (2-4). The preoperative patellofemoral pain scores and 3-month postoperative scores were compared between the experimental and positive control groups, and found that pain was significantly relieved following the resection of the symptomatic MP.

The distribution of SP nerve fiber density in MP and the pain scores of patients with patellofemoral pain were analyzed. The same conclusion could be drawn from contrastive analysis based on each group on nerve fiber density and pain scores: SP expression nerve fibers increased significantly in the patients with symptomatic MP, and its abnormal structure was closely associated with the patients who reported an aggravation of patellofemoral pain.

Considering that the patients with symptomatic MP had cartilage injuries in patellofemoral joint in the majority of cases, the patients were divided into two groups so that the influence of cartilage injuries could be avoided. No significant difference in preoperative pain score was observed between the two groups. With regards to the number of nerve distribution between the two groups, the positive control group was higher compared to that of the experimental group. This may be attributed to the presence of the patellofemoral joint cartilage injury, with loss of cartilage debris entering the patellar medial synovial tissue, synovial cells 
breaking the metabolic balance of local materials, causing local inflammation in the synovial tissue (24), and affecting the local neural structures. Wu et al (25) in 2000 confirmed that the nerve fiber density increased in the ankle osteoarthritis inflammatory synovial tissues, and only a small amount of nerve distribution existed in the healthy synovial tissue.

The present study has two limitations. Firstly, the blank control group was the patients with lateral discoid meniscus. The tear of lateral meniscus and the debris of cartilage may deduce the inflammation in the synovial membrane. The density of the SP fibers in MP may be higher compared to that of normal people. By contrast, semi-quantitative analysis of innervation for SP fibers was variable, possibly implying the trend of SP fibers distribution, however, it was not precise enough to evaluate the number of SP fibers innervated in the plica. Quantitative analysis of the density may improve this.

In conclusion, an increase of SP expression in symptomatic MP may be the important pathophysiological basis that causes pain. The density of SP expression nerve fibers and the level of pain are closely associated. Expression of SP has an important role in the pathogenic mechanisms of symptomatic MP.

\section{References}

1. Dupont JY: Synovial plicae of the knee. Controversies and review. Clin Sports Med 16: 87-122, 1997.

2. Kerimoğlu S, Citlak A, Cavuşoğlu S and Turhan AU: Bucket-handle tear of medial plica. Knee 12: 239-241, 2005.

3. Madhusudhan TR, Kumar TM, Bastawrous SS and Sinha A: Clinical examination, MRI and arthroscopy in meniscal and ligamentous knee Injuries - a prospective study. J Orthop Surg 3: 19, 2008.

4. Boyd CR, Eakin C and Matheson GO: Infrapatellar plica as a cause of anterior knee pain. Clin J Sport Med 15: 98-103, 2005.

5. Wojtys EM, Beaman DN, Glover RA and Janda D: Innervation of the human knee joint by substance-P fibers. Arthroscopy 6 : 254-263, 1990

6. Inami S, Shiga T, Tsujino A, Yabuki T, Okado N and Ochiai N: Immunohistochemical demonstration of nerve fibers in the synovial fold of the human cervical facet joint. J Orthop Res 19: 593-596, 2001.

7. Witoński D and Wagrowska-Danielewicz M: Distribution of substance-P nerve fibers in the knee joint in patients with anterior knee pain syndrome. A preliminary report. Knee Surg Sports Traumatol Arthrosc 7: 177-183, 1999.
8. Ferretti A: Epidemiology of jumper's knee. Sports Med 3: 289-295, 1986.

9. Hardaker WT, Whipple TL and Bassett FH. Diagnosis and treatment of the plica syndrome of the knee. J Bone Joint Surg Am 62: 221-225, 1980.

10. Calpur OU, Copuroglu C and Ozcan M: United unresorbed medial and lateral plicae as anterior mesenchymal synovial septal remnant. Knee Surg Sports Traumatol Arthrosc 10: 378-380, 2002.

11. Farcas C, Hargital Z, Gaspar L, Kuki A, Csernatony Z and Szepesi K: Histological changes in the symptomatic mediopatellar plica. Knee 11: 103-108, 2004.

12. Koshino T and Okamoto R: Resection of painful shelf (plica synovialis mediopatellaris) under arthroscopy. Arthroscopy 1: 136-141, 1985.

13. Sakakibara J: Arthroscopic study on lino's band (plicasynovia is mediopatellaris). J Jpn Orthop Assoc 50: 513-522, 1976.

14. Munzinger U, Ruckstuhl J, Scherrer H and Gschwend N: Internal derangement of the knee joint due to pathologic synovial folds: The mediopatellar plica syndrome. Clin Orthop Relat Res 155: 59-64, 1981.

15. Broom MJ and Fulkerson JP: The plica syndrome: A new perspective. Orthop Clin North Am 17: 279-281, 1986.

16. Jackson RW, Marshall DJ and Fujisawa Y: The pathologic medical shelf. Orthop Clin North Am 13: 307-312, 1982.

17. Shahriaree H and Nottage WM: Synovial folds and plicae. In: O'Connor Textbook of Arthroscopic Surgery. 2nd edition. Shahriaree H (ed). JB Lippincott, Philadelphia, pp535-541, 1992.

18. Biedert RM and Sanchis-Alfonso V: Sources of anterior knee pain. Clin Sports Med 21: 335-347, 2002.

19. Sanchis-Alfonso V and Roselló-Sastre E: Anterior knee pain in the young patient - what causes the pain? 'Neural model.' Acta Orthop Scand 74: 697-703, 2003.

20. Dragoo JL, Johnson C and McConnell J: Evaluation and treatment of disorders of the infrapatellar fat pad. Sports Med 42: 51-67, 2012.

21. Sanchis-Alfonso V: Patellofemoral pain. Orthopade 37: 835-836, 838-840, 2008 (In German).

22. Ordeberg G: Characterization of joint pain in human OA. Novartis Found Symp 260: 105-115, discussion 115-121, 277-279, 2004.

23. Seidel MF and Lane NE: Control of arthritis pain with anti-nerve-growth factor: Risk and benefit. Curr Rheumatol Rep 14: 583-588, 2012.

24. Lyu SR, Tzeng JE, Kuo CY, Jian AR and Liu DS: Mechanical strength of mediopatellar plica - the influence of its fiber content. Clin Biomech (Bristol, Avon) 21: 860-863, 2006.

25. Wu Z, Nagata K and Iijima T: Immunohistochemical study of NGF and its receptors in the synovial membrane of the ankle joint of adjuvant-induced arthritic rats. Histochem Cell Biol 114: $453-459,2000$ 